\title{
THE SOLUTION OF A NONLINEAR GRONWALL INEQUALITY
}

\author{
BURRELL W. HELTON
}

Abstract. This paper extends some of the earlier results of $\mathbf{J}$. V. Herod, W. W. Schmaedeke and G. R. Sell, and B. W. Helton and shows that, under the given conditions,

(1) there is a function $u$ satisfying the inequality

$$
f(x) \leqq h(x)+(R L) \int_{a}^{x}(f G+f H)
$$

such that, if $f$ satisfies the given inequality, then $f(x) \leqq u(x)$; and

(2) there is a function $u$ satisfying the inequality

$$
0<f(x) \leqq k+(R L) \int_{a}^{x}\left[\left(f G_{1}+f^{p n} G_{2}\right)+\left(f H_{1}+f^{p n} H_{2}\right)\right],
$$

where $n$ is a positive integer and $p= \pm 1$ and $p n \neq 1$, such that, if $f$ satisfies the given inequality, then $f(x) \leqq u(x)$.

Definitions AND NOtations. $R$ is the set of real numbers: capital letters and lower case letters denote functions from $R \times R$ to $R$ and from $R$ to $R$, respectively; $f^{-1}=1 / f$; all integrals are subdivision-refinement type limits; $(L R) \int_{a}^{b}(f G+g H) \sim f\left(x_{i-1}\right) G\left(x_{i-1}, x_{i}\right)+g\left(x_{i}\right) H\left(x_{i-1}, x_{i}\right) ; G \in O A^{\circ}$ on $[a, b] \Rightarrow \int_{a}^{b} G$ exists and $\int_{a}^{b}\left|G-\int G\right|=0 ; G \in O M^{\circ}$ on $[a, b] \Rightarrow_{x} \Pi^{y}(1+G)$ exists for $a \leqq x<y \leqq b$ and $\int_{a}^{b}|(1+G)-\Pi(1+G)|=0 ; G \in O B^{\circ} \Rightarrow$ bounded variation; $H \geqq 0,(1-G)^{-1}$ exists, $G \in O B^{\circ}$, etc. means there is a subdivision $\left\{x_{i}\right\}_{0}^{n}$ of $[a, b]$ such that if $0<i \leqq n$, then, on $\left[x_{i-1}, x_{i}\right], H \geqq 0$, $(1-G)^{-1}$ exists, $G$ has bounded variation, etc., respectively; $G \in O L^{\circ}$ on $[a, b]$ means the limits $G\left(y^{-}, y^{-}\right), G\left(x^{+}, x^{+}\right), G\left(y^{-}, y\right)$ and $G\left(x, x^{+}\right)$exist for $a \leqq x<y \leqq b$. For detailed definitions see [1] and [2].

Lemma 1. If $G \in O A^{\circ}$ and $O B^{\circ}$ on $[a, b]$, then $G \in O L^{\circ}$ on $[a, b]$.

LeMma 2. If $\int_{a}^{b} G^{2}=0, \int_{a}^{b} H^{2}=0$ and $f$ is a bounded function, then $\int_{a}^{b} G H=0$ and $\int_{a}^{b} f G^{2}=0$.

Received by the editors September 9, 1971 and, in revised form, July 17, 1972.

AMS (MOS) classifications (1970). Primary 26A86; Secondary 26A39, 26A42, $45 \mathrm{G} 99$.

Key words and phrases. Integrals, product integrals, Gronwall inequality, integral equations.

(c) American Mathematical Society 1973 
LEMMA 3. If $H$ and $G$ are functions from $R \times R$ to $R$ such that $H \in O L^{\circ}$ and $G \in O A^{\circ}$ and $O B^{\circ}$ on $[a, b]$ then $H G \in O A^{\circ}$ and $O M^{\circ}$ on $[a, b][2$, Theorem 2].

LEMMA 4. If $f$ is a function from $R$ to $R$ and $H$ and $G$ are functions from $R \times R$ to $R$ such that on $[a, b] f$ has bounded variation, $H$ and $G \in O A^{\circ}$ and $O B^{\circ}$, and $(1-G)^{-1}$ exists and is bounded, then on $[a, b],(1-G)^{-1} \in O L^{\circ}$, and each of $d f(1-G)^{-1}, H(1-G)^{-1}, f(x) H(x, y), f(y) H(x, y)$ and $(1+H)$. $(1-G)^{-1}-1=(H+G)(1-G)^{-1}$ belongs to $O A^{\circ}$ and $O M^{\circ}$.

Lemma 4 is a corollary to Lemma 3.

LEMMA 5. If $f$ is quasicontinuous and $G \in O A^{\circ}$ and $O B^{\circ}$ on $[a, b]$, then $f G \in O A^{\circ}$ and $O B^{\circ}$ on $[a, b]$.

Lemma 5 is a corollary to Lemma 3.

LEMMA 6. Given. $H$ and $G$ are functions from $R \times R$ to $R, c \in R$ and $c>0 ; H$ and $G \in O A^{\circ}$ and $O B^{\circ}, H \geqq 0, G \geqq 0$ and $1-G \geqq c$ on $[a, b]$; and $u$ is a function from $R$ to $R$ such that $u$ is bounded above on $[a, b]$, $(L R) \int_{a}^{b}(u H+u G)$ exists and $u(x) \leqq(L R) \int_{a}^{x}(u H+u G)$ for $x \in[a, b]$.

Conclusion. If $x \in[a, b]$, then $u(x) \leqq 0[2$, Theorem 3].

LEMMA 7. Given. $f$ and $h$ are functions from $R$ to $R$ and $H, G$ and $B$ are functions from $R \times R$ to $R$ such that $f(a)=h(a), h$ has bounded variation, $(1-G)^{-1}$ exists and is bounded, dh $(1-G)^{-1} \in O A^{\circ}, B=(1+H)(1-G)^{-1}$ and $B-1 \in O B^{\circ}$ and $O M^{\circ}$ on $[a, b]$.

Conclusion. The following statements are equivalent:

1. $(L R) \int_{a}^{b}(f H+f G)$ exists and $f(x)=h(x)+(L R) \int_{a}^{x}(f H+f G)$ for $x \in[a, b]$; and

2. if $a<x \leqq b$, then $(L) \int_{a}^{x}\left|f(t)\left[B-\prod B\right]\right|=0$ and

$$
f(x)=f(a)_{a} \prod^{x} B+(R) \int_{a}^{x} d h(1-G)^{-1} \prod_{t}^{x} B .
$$

Lemma 7 is a special case of Theorem 5.1 [1, p. 310].

LEMMA 8. If $G$ is a function from $R \times R$ to $R$ and $G \in O B^{\circ}$, then the following statements are equivalent: (1) $G \in O A^{\circ}$ and (2) $G \in O M^{\circ}[1$, Theorem 3.4].

LEMMA 9. If $[a, b]$ is a number interval and $F$ and $G$ are functions from $R \times R$ to $R$ such that $\int_{a}^{b}|F G|=0, \int_{a}^{b} G^{2}=0$, and $F$ and $G \in O B^{\circ}$ and $O M^{\circ}$ on $[a, b]$, then

(1) ${ }_{a} \Pi^{b}(1+F)_{a} \Pi^{b}(1+G)={ }_{a} \Pi^{b}(1+F+G)$;

(2) if $n$ is a positive integer, then $\left[a \Pi^{b}(1+G)\right]^{n}={ }_{a} \Pi^{b}(1+n G)$; and

(3) if $\Pi_{a} \Pi^{b}(1-G)$ exists, then $\left[\Pi_{a}^{b}(1+G)\right]^{-1}={ }_{a} \Pi^{b}(1-G)$. 
Lemma 9 is a special case of Theorem 5.6 [1, p. 315].

LEMMA 10. If $G$ is a function from $R \times R$ to $R$ such that $\int_{a}^{b} G$ exists, then $G \in O A^{\circ}$ on $[a, b][1$, Theorem 4.1].

THEOREM 1. Given. $c \in R$ and $c>0 ; H$ and $G$ are functions from $R \times R$ to $R$ such that $H$ and $G \in O B^{\circ}$ and $O A^{\circ}, H \geqq 0, G \geqq 0$ and $1-G \geqq c$ on $[a, b] ; f$ and $h$ are functions from $R$ to $R$ such that $f$ is bounded and $h$ has bounded variation on $[a, b],(L R) \int_{a}^{b}(f H+f G)$ exists and, if $x \in[a, b]$, then

$$
f(x) \leqq h(x)+(L R) \int_{a}^{x}(f H+f G) .
$$

Conclusion. (1) If $B=(1+H)(1-G)^{-1}$, then the function

$$
u(x)=h(a)_{a} \prod^{x} B+(R) \int_{a}^{x} d h(1-G)^{-1} \prod_{t} \prod_{B}
$$

exists on $[a, b]$ and satisfies the inequality in the hypothesis.

(2) If $x \in[a, b]$, then $f(x) \leqq u(x)$.

Proof. If $a \leqq x<y \leqq b$, then ${ }_{x} \Pi^{y} B$ exists and $B-1 \in O M^{\circ}$ (Lemma 4) and $B-1 \in O B^{\circ}$. Since $G \in O A^{\circ}$ and $O B^{\circ}$ and $1-G \geqq c>0$, then $G, 1-G$ and $(1-G)^{-1} \in O L^{\circ}$; hence, $d h(1-G)^{-1} \in O A^{\circ}$ on $[a, x]$ (by Lemma 3 ). Since $B-1 \in O B^{\circ}$, it follows that, for each $x \in[a, b]$, the function $\prod_{t} \prod^{x} B$ has bounded variation and is quasicontinuous on $[a, x]$; since $d h(1-G)^{-1} \in$ $O A^{\circ}$ and $O B^{\circ}$ on $[a, x]$, it follows from Lemma 5 that $d h(1-G)^{-1} \prod_{t} \prod^{x} B \in$ $O A^{\circ}$ on $[a, x]$. Let $u$ be the function such that, if $x \in[a, b]$, then

$$
u(x)=h(a)_{a} \prod^{x} B+(R) \int_{a}^{x} d h(1-G)^{-1}+\prod^{x} B .
$$

It follows from Lemma 7 that, if $x \in[a, b]$, then

$$
u(x)=h(x)+(L R) \int_{a}^{x}(u H+u G)
$$

and $f(x)-u(x) \leqq(L R) \int_{a}^{x}[(f-u) H+(f-u) G]$ and, from Lemma 6, that $f(x)-u(x) \leqq 0$.

Theorem 4 in [2] is a special case of the above theorem. If $f$ has bounded variation, the above inequality is a special case of the one given by Herod [3, Remark, p. 36]; however, Theorem 1 gives the best upper bound of the solution set for this inequality and this type of integral.

THEOREM 2. Given. (1) $[a, b]$ is a number interval, $n$ is a positive integer, $k>0, p=1$ or $p=-1$, and $p n \neq 1$; (2) $G_{1}, G_{2}, H_{1}, H_{2}$ are functions from $R \times R$ to $R$ and each belongs to $O A^{\circ}$ and $O B^{\circ}, G_{1} \geqq 0, H_{1} \geqq 0, p G_{2} \geqq 0$ and $p H_{2} \geqq 0$ on $[a, b]$ and $\int_{a}^{b} G_{1}^{2}=\int_{a}^{b} G_{2}^{2}=\int_{a}^{b} H_{1}^{2}=\int_{a}^{b} H_{2}^{2}=0$; and (3) $f$ is a bounded 
function from $R$ to $R$ such that $f$ is bounded away from zero on $[a, b]$ and, if $x \in[a, b]$, then $(L) \int_{a}^{x} f^{p m} G_{2}$ and $(R) \int_{a}^{x} f^{p m} H_{2}$ exist for $m=1,2, \cdots, n$, and the following integral exists, and

$$
0<f(x) \leqq k+(L R) \int_{a}^{x}\left[\left(f G_{1}+f^{p n} G_{2}\right)+\left(f H_{1}+f^{p n} H_{2}\right)\right] .
$$

Conclusion. (1) If $x \in[a, b]$, then $g(x)={ }_{a} \Pi^{x}\left(1+G_{1}+H_{1}\right)$ and (LR) $\int_{a}^{x}\left(g^{p n-1} G_{2}+g^{p n-1} H_{2}\right)$ exist. (2) If $u$ and $h$ are functions from $R$ to $R$ such that, on $[a, b]$,

and

$$
h(x)=k^{1-p n}+(1-p n)(L R) \int_{a}^{x}\left(g^{p n-1} G_{2}+g^{p n-1} H_{2}\right)>0
$$

$$
u(x)=g(x) h(x)^{1 /(1-p n)},
$$

then $f(x) \leqq u(x)$ for $x \in[a, b]$. Furthermore, on $[a, b]$ the function $u$ is bounded away from zero and satisfies the inequality stated in the hypothesis.

Proof. Since $G_{1}$ and $H_{1} \in O A^{\circ}$ and $O B^{\circ}$ on $[a, b]$, then $G_{1}+H_{1} \in O A^{\circ}$ and $O B^{\circ}$ and, by Lemmas 1 and $8, G_{1}+H_{1} \in O M^{\circ}$ and $O L^{\circ}$ and $-\left(G_{1}+H_{1}\right)$ $\in O B^{\circ}, O A^{\circ}, O L^{\circ}$ and $O M^{\circ}$ on $[a, b]$; hence, $g(x)={ }_{a} \Pi^{x}\left(1+G_{1}+H_{1}\right)$ exists for $x \in[a, b]$ and, by Lemma $9, g^{-1}(x)={ }_{a} \Pi^{x}\left[1-\left(G_{1}+H_{1}\right)\right]$ for $x \in[a, b]$. If $a \leqq x<y \leqq b$, then there is a number $e$ such that

$$
\begin{aligned}
g(y)-g(x) & ={ }_{a} \prod^{y}\left(1+G_{1}+H_{1}\right)-{ }_{a} \prod^{x}\left(1+G_{1}+H_{1}\right) \\
& =\prod_{a}^{x}\left(1+G_{1}+H_{1}\right)\left[\prod_{x} \prod^{y}\left(1+G_{1}+H_{1}\right)-1\right] \\
& ={ }_{a} \prod^{x}\left(1+G_{1}+H_{1}\right)\left[G_{1}(x, y)+H_{1}(x, y)+e\right] .
\end{aligned}
$$

Therefore, since $G_{1}+H_{1} \in O M^{\circ}$ and since $\int_{a}^{b} G_{1}^{2}=0$ and $\int_{a}^{b} H_{1}^{2}=0$, then $g$ is continuous on $[a, b]$ and, since $G_{1}+H_{1} \in O B^{\circ}$, then $g$ has bounded variation on $[a, b]$. Similarly, $g^{-1}$ is continuous and has bounded variation on $[a, b]$. Since $G_{2}$ and $H_{2} \in O A^{\circ}$ and $O B^{\circ}$ and since $g$ and $g^{-1}$ are continuous on $[a, b]$, then $(L R) \int_{a}^{b}\left(g^{p n-1} G_{2}+g^{p n-1} H_{2}\right)$ exists (Lemma 5). Since $\int_{a}^{b} G_{2}^{2}=\int_{a}^{b} H_{2}^{2}=0$, then $h$ is continuous on $[a, b]$.

Let $u$ be the function such that $u(x)=g(x) h(x)^{1 /(1-p n)}$ on $[a, b]$; then $u$ and $u^{-1}$ exist and are continuous and, if $x \in[a, b]$, then

$$
\begin{aligned}
u(x)^{1-p n} g(x)^{p n-1}= & h(x) \\
= & k^{1-p n}+(L R) \int_{a}^{x}\left\{\left(u^{1-p n} g^{p n-1}\right)\left[u^{p n-1}(1-p n) G_{2}\right]\right. \\
\left.+\left(u^{1-p n} g^{p n-1}\right)\left[u^{p n-1}(1-p n) H_{2}\right]\right\} & \\
= & k^{1-p n}(L R)_{a} \prod^{x}\left\{1+\left[u^{p n-1}(1-p n) G_{2}\right]\right\} \\
& \cdot\left\{1-\left[u^{p n-1}(1-p n) H_{2}\right]\right\}^{-1},
\end{aligned}
$$


by Lemma 7 . Hence, if $x \in[a, b]$, then

and

$$
u(x)=g(x) k(L R)_{a} \prod^{x}\left(1+u^{p n-1} G_{2}\right)\left(1-u^{p n-1} H_{2}\right)^{-1}
$$

$$
g(x)={ }_{a} \Pi^{x}\left(1+G_{1}+H_{1}\right)={ }_{a} \prod^{x}\left(1+G_{1}\right)_{a} \prod^{x}\left(1+H_{1}\right)
$$

(Lemmas 8, 9)

and

$$
u(x)=k(L R)_{a} \prod^{x}\left[1+\left(G_{1}+u^{p n-1} G_{2}\right)\right]\left[1-\left(H_{1}+u^{p n-1} H_{2}\right)\right]^{-1}
$$

(Lemma 9).

Therefore, if $x \in[a, b]$, then

Hence,

$$
\begin{aligned}
u(x) & =k+(L R) \int_{a}^{x}\left[u\left(G_{1}+u^{p n-1} G_{2}\right)+u\left(H_{1}+u^{p n-1} H_{2}\right)\right] \text { (Lemma 7) } \\
& =k+(L R) \int_{a}^{x}\left[\left(u G_{1}+u^{p n} G_{2}\right)+\left(u H_{1}+u^{p n} H_{2}\right)\right] .
\end{aligned}
$$

$$
\begin{aligned}
f(x)-u(x) & \leqq(L R) \int_{a}^{x}\left\{\left[(f-u) G_{1}+\left(f^{p n}-u^{p n}\right) G_{2}\right]\right. \\
& \left.+\left[(f-u) H_{1}+\left(f^{p n}-u^{p n}\right) H_{2}\right]\right\} \\
& =(L R) \int_{a}^{x}\left[(f-u)\left(G_{1}+v G_{2}\right)+(f-u)\left(H_{1}+v H_{2}\right)\right]
\end{aligned}
$$

where

$$
v=p\left(f^{n-1}+f^{n-2} u+\cdots+u^{n-1}\right)\left(f^{-n} u^{-n}\right)^{(1-p) / 2} .
$$

Since $f, f^{-1}, u$ and $u^{-1}$ are bounded on $[a, b]$, then $v$ is bounded on $[a, b]$ and, since $\int_{a}^{b} H_{1}^{2}=\int_{a}^{b} H_{2}^{2}=0$, then it follows from Lemma 2 that $\int_{a}^{b}\left(H_{1}+v H_{2}\right)^{2}=0$ and hence $1-\left(H_{1}+v H_{2}\right) \geqq \frac{1}{2}$. It follows from the hypothesis that (R) $\int_{a}^{b} f^{r} H_{2}$ exists and $f^{r} H_{2} \in O B^{\circ}$ for $r=0, \pm 1, \pm 2, \cdots, \pm n$ and that $f^{r} H_{2} \in O A^{\circ}$ (Lemma 10). Since $u$ and $u^{-1}$ are continuous, then

$$
\text { (R) } \int_{a}^{b} f^{n-r} u^{r-1}\left(f^{-n} u^{-n}\right)^{(1-p) / 2} H_{2}
$$

exists for $r=1,2, \cdots, n-1$ and $p= \pm 1$ (Lemma 5). Therefore, $(R) \int_{a}^{b} v H_{2}$ and $(R) \int_{a}^{b}\left(H_{1}+v H_{2}\right)$ exist and $H_{1}+v H_{2} \in O A^{\circ}$ (Lemma 10). Similarly, $G_{1}+v G_{2} \in O A^{\circ}$. Since $f \geqq 0$ and $u \geqq 0$ on $[a, b]$, then $p v \geqq 0$ and it follows from the hypothesis that $G_{1}+v G_{2}$ and $H_{1}+v H_{2}$ are nonnegative on $[a, b]$. It follows from Lemma 6 that $f-u \leqq 0$ on $[a, b]$.

NoTE 1. If $p=1$, then the requirement that $f$ be bounded away from zero may be deleted.

Note 2. If $p n=0$ or $p n=1$, then Theorem 2 can be simplified to a special case of Theorem 1. 
Note 3. If $f^{p m}$ is quasicontinuous, then the integrals in part (3) of the hypothesis will exist.

EXAMPLE. Suppose that $f$ and $h$ are functions from $R$ to $R$ such that, on $[a, b], h$ is continuous, $h \geqq 0, f(a) \geqq 1, f$ is bounded and bounded away from zero, $f^{\prime}$ exists and is continuous, and $f^{\prime}(x) \leqq\left[f(x)-f^{-2}(x)\right] h(x)$; then

$$
f(x) \leqq f(a)+\int_{a}^{x}\left[f h d t-f^{-2} h d t\right] .
$$

The hypothesis of Theorem 2 is satisfied with $G_{1}=h d t, G_{2}=-h d t, H_{1}=$ $H_{2}=0, n=2$ and $p=-1$. Hence, if $x \in[a, b]$, then

and

$$
g(x)=\prod_{a}^{x}(1+h d t)=\exp \int_{a}^{x} h d t,
$$

$$
\begin{aligned}
f(x) & \leqq u(x) \\
& =\left(\exp \int_{a}^{x} h d t\right)\left\{f(a)^{3}+3(L) \int_{a}^{x}\left[\left(\exp \int_{a}^{t} h d q\right)^{-3}(-h d t)\right]\right\}^{1 / 3} \\
& =\left\{\left(\exp 3 \int_{a}^{x} h d t\right)\left[f(a)^{3}-1\right]+1\right\}^{1 / 3} .
\end{aligned}
$$

If $0 \leqq a<b$ and $f$ and $h$ are functions such that $f(x)=e^{x}\left(1+e^{-3 x}\right)^{1 / 3}$ and $h(x)=1+x$ for $x \in[a, b]$, then $f$ and $h$ satisfy the hypothesis of the above example.

\section{BIBLIOGRAPHY}

1. B. W. Helton, Integral equations and product integrals, Pacific J. Math. 16 (1966), 297-322. MR 32 \#6167.

2. - A product integral representation for a Gronwall inequality, Proc. Amer. Math. Soc. 23 (1969), 493-500. MR 40 \#1562.

3. J. V. Herod, A Gronwall inequality for linear Stieltjes integrals, Proc. Amer. Math. Soc. 23 (1969), 34-36. MR 40 \#2802.

4. W. W. Schmaedeke and G. R. Sell, The Gronwall inequality for modified Stieltjes integrals, Proc. Amer. Math. Soc. 19 (1968), 1217-1222. MR 37 \#6422.

Department of Mathematics, Southwest Texas State University, San Marcos, TEXAS 78666 